\title{
Case Report Hydrogen Peroxide Induced Colitis: A Case Report and Literature Review
}

\author{
Dushyant Pawar, ${ }^{1}$ Anna Calara, ${ }^{1}$ Roy Jacob, ${ }^{2}$ Nancy Beck, ${ }^{3}$ and Alan N. Peiris ${ }^{4}$ \\ ${ }^{1}$ School of Medicine, Texas Tech University Health Sciences Center, Lubbock, TX, USA \\ ${ }^{2}$ Department of Radiology, University Medical Center, Lubbock, TX, USA \\ ${ }^{3}$ Department of Pediatrics, Texas Tech University Health Sciences Center, Lubbock, TX, USA \\ ${ }^{4}$ Department of Internal Medicine, Texas Tech University Health Sciences Center, Lubbock, TX, USA
}

Correspondence should be addressed to Dushyant Pawar; dushyant.s.pawar@ttuhsc.edu

Received 9 October 2017; Accepted 4 December 2017; Published 25 December 2017

Academic Editor: Tetsuo Hirata

Copyright (C) 2017 Dushyant Pawar et al. This is an open access article distributed under the Creative Commons Attribution License, which permits unrestricted use, distribution, and reproduction in any medium, provided the original work is properly cited.

\begin{abstract}
Constipation is a common condition. Hydrogen peroxide enemas have rarely been reported as a home remedy for constipation in the pediatric age group. We present a case report and literature review of hydrogen peroxide induced colitis in pediatric siblings, aged 2 years and 9 years. The siblings presented with vomiting and bloody diarrhea an hour following the enema. Physical exam, vital signs, blood, and electrolyte counts were normal, but CT scans showed mucosal thickening of the rectum and colon. Their symptoms resolved after oral intake was curtailed and fluids were replaced intravenously. We discuss existing reports of complications from hydrogen peroxide enemas. Patients may present with abdominal pain and bloody diarrhea. Onset of symptoms varied from minutes to a day and bowel ulceration with necrosis and perforation has occurred, although fatality is rare. Diagnostic tests included computed tomography (CT) scan, sigmoidoscopy, or biopsy. Recovery period ranged from 3 days to 8 months. Public education regarding the dangers of hydrogen peroxide enemas is needed.
\end{abstract}

\section{Introduction}

Constipation is a common problem, reported in up to $30 \%$ of children. Home treatment for constipation includes eating foods high in fiber such as vegetables, citrus fruits, beans, and whole grains. A healthcare provider should be consulted before giving a laxative or enema to children. Internet searches for homemade remedies to treat constipation deliver hydrogen peroxide enema among the results. Hydrogen peroxide has been known to cause colitis and, in a few cases, bowel perforation. The earliest use of hydrogen peroxide enema in the pediatric population is documented by Olim and Ciuti to treat meconium ileus in the newborn [1].

Herein we present an unusual case of two siblings, aged 2 years and 9 years, presenting with bloody diarrhea and vomiting due to chemical colitis following hydrogen peroxide enema. We also present a systematic review of prior such cases. Few such cases have been reported in the pediatric population.

\section{Case Presentation}

Two siblings, a 2-year-old male and a 9-year-old female, had been constipated for 2 days. Patients did not have a family history of gastrointestinal issues and past medical history was unremarkable. The mother of the patients was providing both children with a regular but meat-free soy-based diet of her own volition. However, the children had rock-hard stools that could not be passed. Both patients were not given any prior conventional or homemade remedies. However, following an Internet search, both patients were then given an enema of hydrogen peroxide mixed with warm water. Concentration of hydrogen peroxide used is unknown. Following the enema, the patients passed stools but, an hour later, started vomiting and had bloody diarrhea.

Upon admission, vital signs were normal. Both patients had soft abdomens on exam, which were nontender to deep palpation, nondistended, and no guarding was noted. Physical exam did not raise concerns for perforations and the 


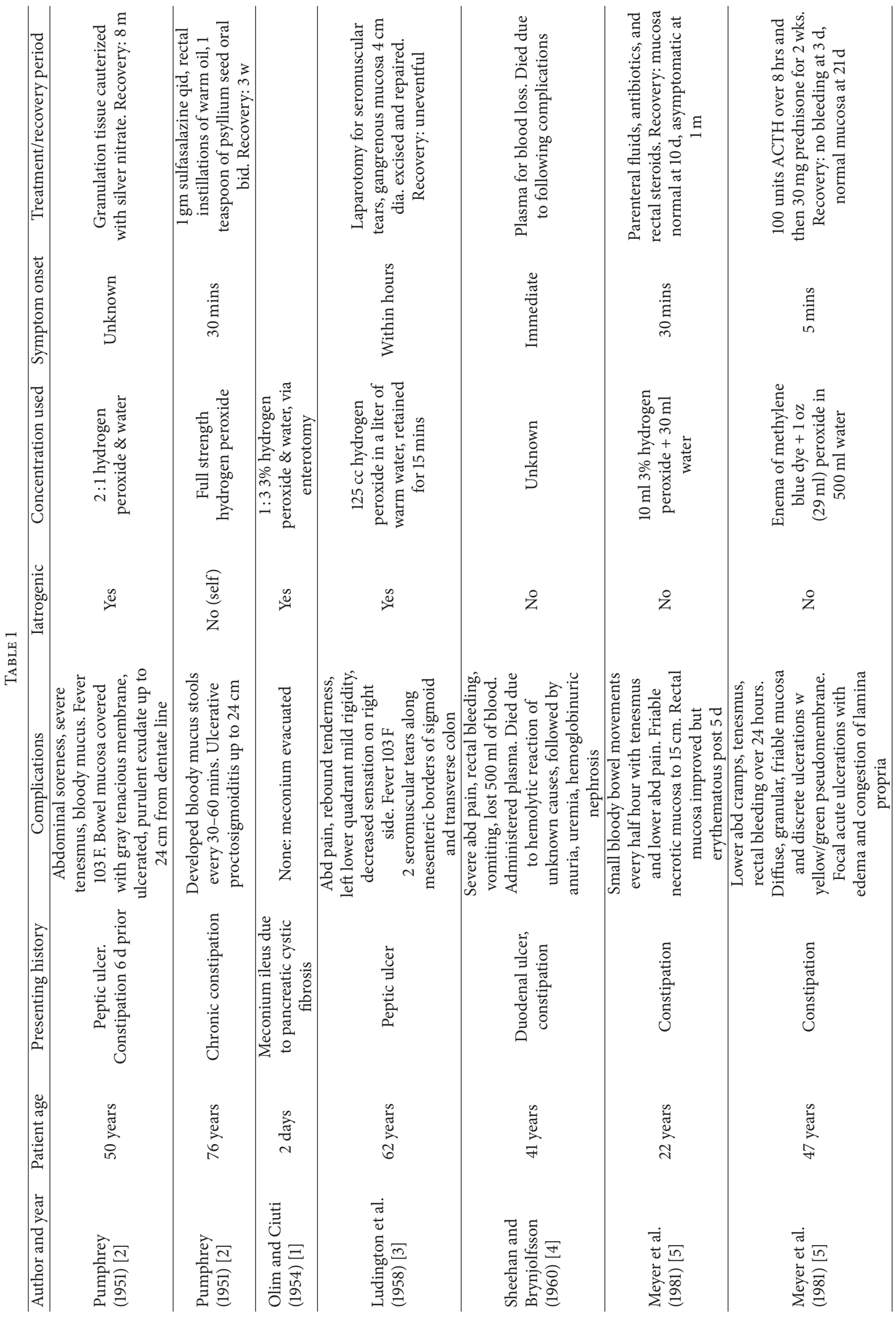




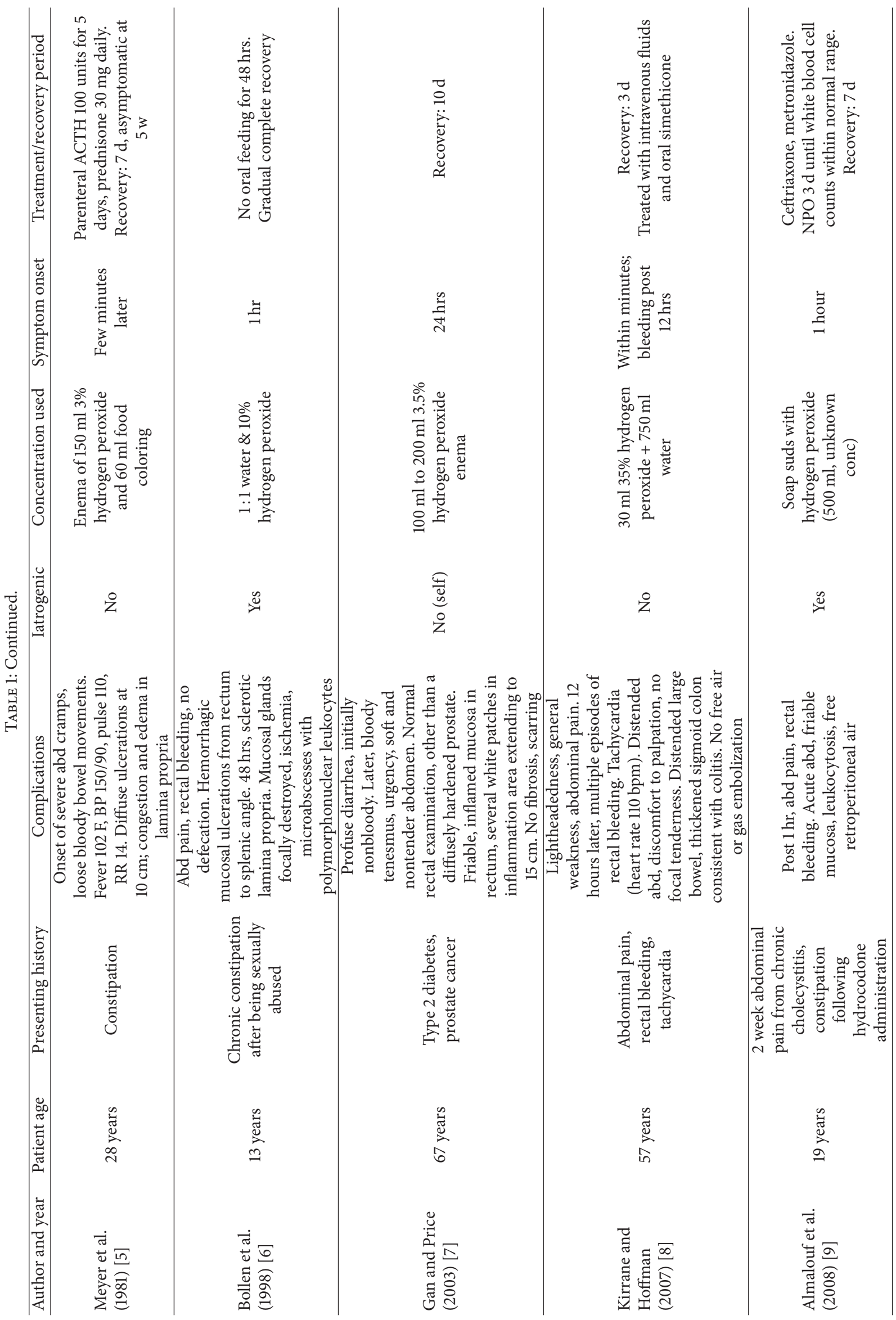




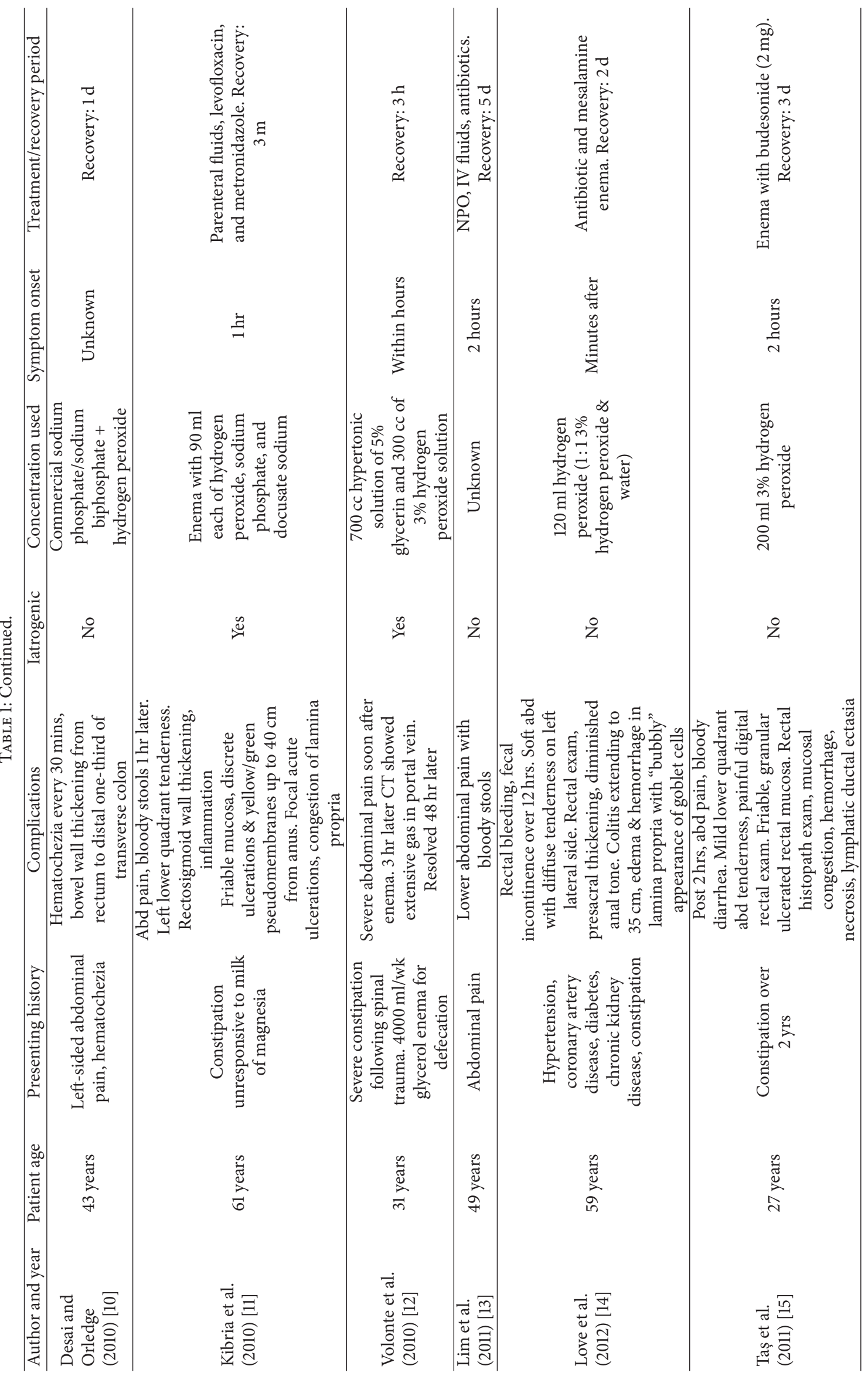


patients denied abdominal pain. Complete blood count and metabolic panel for both patients were within normal limits.

Abdominal CT for the patient of age 2 years showed mucosal thickening of the rectum and sigmoid colon with no free air. The patient aged 9 years had a more extensive mucosal thickening of the rectum, sigmoid, and descending colon but showed no perforation or free fluid. Both patients were admitted to the pediatric intensive care unit, had their oral intake withheld, and were given IV fluids. They showed clinical improvement the same day and were then transferred to the pediatric ward. Both patients were discharged 24 hours later with resolution of emesis and bloody stool. They were prescribed polyethylene glycol laxative, 17 grams, daily for constipation.

\section{Discussion}

We found 21 published cases related to hydrogen peroxide enema. Prior cases show the patient population as mostly older adults with constipation from secondary causes. To our knowledge, this is the first case of colitis due to hydrogen peroxide enema in siblings in the pediatric age group. The most recent case report was published in 2016 [16]. Cases related to oral ingestion of hydrogen peroxide as well as those associated with contamination during endoscopic examinations were excluded.

In 11 of the 21 cases, enema was self-administered; in the rest, enema was administered under physician supervision. The enema was administered to treat constipation, except for one case where the patient used it to treat prostate cancer. The enema concentrations varied and are listed in Table 1. Following the enema, patients were able to relieve their constipation but then presented with symptoms of abdominal pain/tenderness, bloody diarrhea, fever, tenesmus, leukocytosis, and/or tachycardia [17]. Endoscopy findings included mucosal friability, exudates, ulceration, necrosis, and/or perforation of the distal colon or rectum (Table 1) [17]. The onset of bloody diarrhea after administration of hydrogen peroxide enema varied from a few minutes to an entire day following the enema. Diagnostic tests varied with some combination of CT scan (4 cases), sigmoidoscopy (11 cases), or biopsy (4 cases). In our case, diagnosis was made solely using abdominal CT scan and patient history, without relying on additional sigmoidoscopy and biopsy findings because the temporal relationship of presenting symptoms following the enema favored the diagnosis of hydrogen peroxide induced colitis.

Recovery period ranged from 3 days to 8 months, with mostly uneventful gradual recovery. Recommended treatment included bowel rest, fluid resuscitation, and broad spectrum antibiotics, NSAIDs, or corticosteroids [17]. Depending on the extent of injury, most patients recovered after conservative medical therapy. However, serious consequences included death due to idiopathic hemolytic reaction following plasma transfusion to correct blood loss [4], portal vein embolism [12], and colonic rupture [3]. A summary comparing and contrasting diagnostic tests findings, medications prescribed, and complications of hydrogen peroxide induced colitis are documented in Table 1.
Hydrogen peroxide is available over the counter in concentrations of $3 \%$ and a "food grade" variety of $35 \%$. Although it has a warning label stating "For external use only," a study by the National Survey of Consumers and Health Professionals found that only $7 \%$ of consumers read usage warnings. Hence, public education on the dangers of hydrogen peroxide enema may be needed. It may be effective since patients tend to follow the advice of a competent physician over information obtained from the Internet [18]. Hydrogen peroxide has also been used for other indications like enlarged prostate and cancer of prostate [7]. Therefore, patients with unexplained colitis or proctitis may be queried about use of hydrogen peroxide enema.

Finally, the siblings followed a meat-free soy-based diet, and although soy protein has been known to be an allergen, we did not find an association between soy protein and constipation.

\section{Conflicts of Interest}

The authors declare that there are no conflicts of interest regarding the publication of this article.

\section{Acknowledgments}

The authors wish to acknowledge the contribution of the Texas Tech University Health Sciences Center Clinical Research Institute for their assistance with this research. Funding for article processing charges is provided by Texas Tech University Health Sciences Center Department of Internal Medicine.

\section{References}

[1] C. B. Olim and A. Ciuti, "Meconium ileus: a new method of relieving obstruction; report of two cases with successful management," Annals of Surgery, vol. 140, no. 5, pp. 736-740, 1954.

[2] R. E. Pumphrey, "Hydrogen peroxide proctitis," The American Journal of Surgery, vol. 81, no. 1, pp. 60-62, 1951.

[3] L. G. Ludington, S. W. Hartman, J. E. Keplinger, and F. S. Williams, "Incomplete Rupture of the Colon Following Hydrogen Peroxide Enema," A.M.A Archives of Surgery, vol. 76, no. 4, pp. 658-660, 1958.

[4] J. F. Sheehan and G. Brynjolfsson, "Ulcerative colitis following hydrogen peroxide enema: case report and experimental production with transient emphysema of colonic wall and gas embolism," Laboratory Investigation; A Journal of Technical Methods And Pathology, vol. 9, pp. 150-168, 1960.

[5] C. T. Meyer, M. Brand, V. A. DeLuca, and H. M. Spiro, "Hydrogen peroxide colitis: A report of lhree patients," Journal of Clinical Gastroenterology, vol. 3, no. 1, pp. 31-35, 1981.

[6] P. Bollen, A. Goossens, B. Hauser, and Y. Vandenplas, "Colonic ulcerations caused by an enema containing hydrogen peroxide," Journal of Pediatric Gastroenterology and Nutrition, vol. 26, no. 2, pp. 232-233, 1998.

[7] S. I. Gan and L. M. Price, "Waiting-list induced proctitis: The hydrogen peroxide enema," Canadian Journal of Gastroenterology \& Hepatology, vol. 17, no. 12, pp. 727-729, 2003. 
[8] B. M. Kirrane and R. S. Hoffman, "Abdominal pain and rectal bleeding after an enema," Clinical Toxicology, vol. 45, no. 8, p. 968, 2007.

[9] P. Almalouf, T. M. Shehab, A. M. R. Daniel, E. A. Robinson, and J. L. Barnett, "Therapeutic hydrogen peroxide enema causing severe acute colitis," International Journal of Colorectal Disease, vol. 23, no. 11, pp. 1139-1140, 2008.

[10] Y. Desai and J. Orledge, "Chemical colitis from a hydrogen peroxide enema.", Journal of the Mississippi State Medical Association, vol. 51, no. 11, pp. 314-316, 2010.

[11] R. Kibria, S. A. Ali, and C. J. Barde, "Gone but not forgotten. "bubble gum enema" containing hydrogen peroxide and causing life-threatening colitis," Gastrointestinal Endoscopy, vol. 72, no. 3, pp. 619-620, 2010.

[12] F. Volonte, P. Gervaz, P.-A. Poletti, and P. Morel, "Portal vein gas embolism following oxygen peroxide enema," Colorectal Disease, vol. 12, no. 10, pp. e335-e336, 2010.

[13] C. H. Lim, H. Y. Lee, W. C. Kim et al., "A case of chemical colitis caused by hydrogen peroxide enema," The Korean Journal of Gastroenterology, vol. 58, no. 2, p. 100, 2011.

[14] B. L. Love, D. Pharm, S. Siddiqui, B. J. Mccallum, and R. M. Helman, "Severe chemical colitis due to hydrogen peroxide enema," Journal of Clinical Gastroenterology, vol. 46, no. 1, p. 87, 2012.

[15] A. Taş, Y. Y. Aydin, M. Arhan, and S. Köklü, "Hydrogen peroxide exposure mimicking ulcerative proctitis," Digestive and Liver Disease, vol. 43, no. 4, pp. 331-332, 2011.

[16] J. S. Lee and J. K. Yoo, "Chemical colitis caused by hydrogen peroxide enema in a child: case report and literature review," Journal of Toxicology and Environmental Health, vol. 32, Article ID e2017002, 2016.

[17] S. Sheibani and L. B. Gerson, "Chemical colitis," Journal of Clinical Gastroenterology, vol. 42, no. 2, pp. 115-121, 2008.

[18] J. Laugesen, K. Hassanein, and Y. Yuan, “The impact of internet health information on patient compliance: a research model and an empirical study," Journal of Medical Internet Research, vol. 17, no. 6, article e143, 2015. 


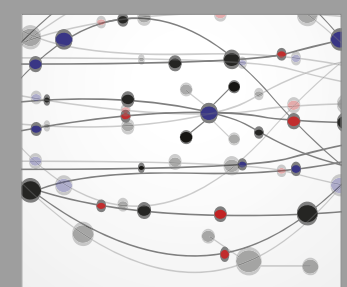

The Scientific World Journal
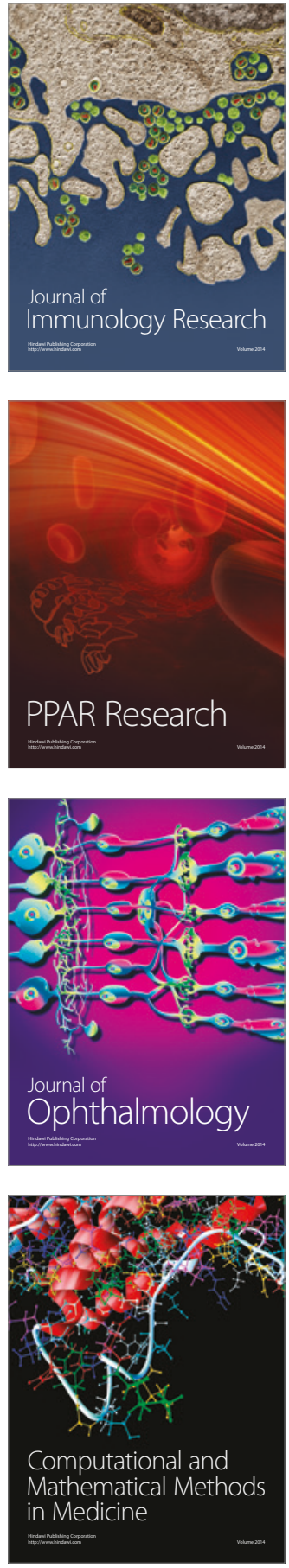

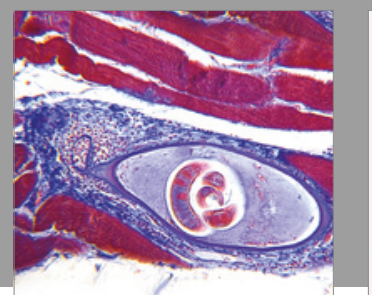

Gastroenterology Research and Practice
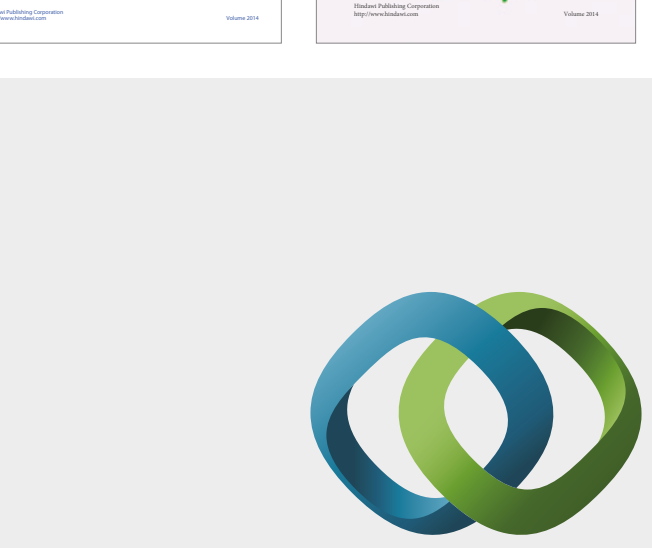

\section{Hindawi}

Submit your manuscripts at

https://www.hindawi.com
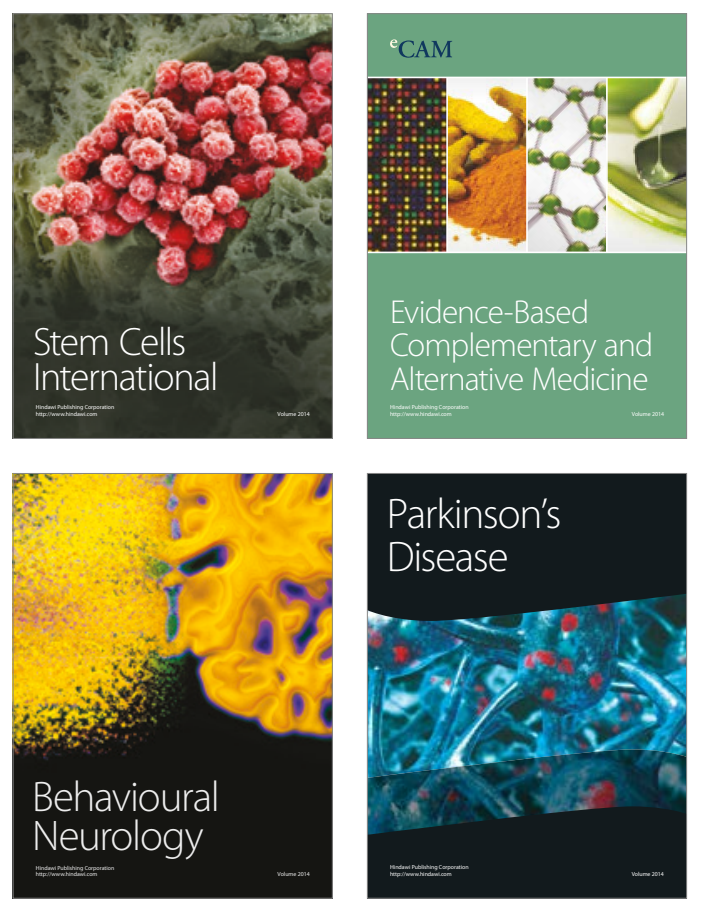
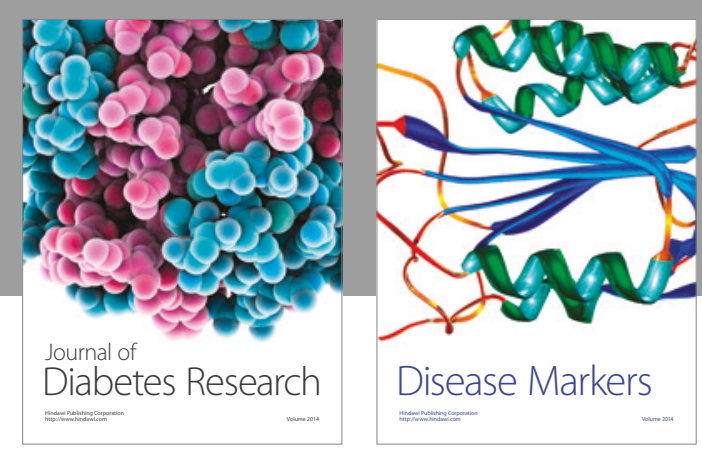

Disease Markers
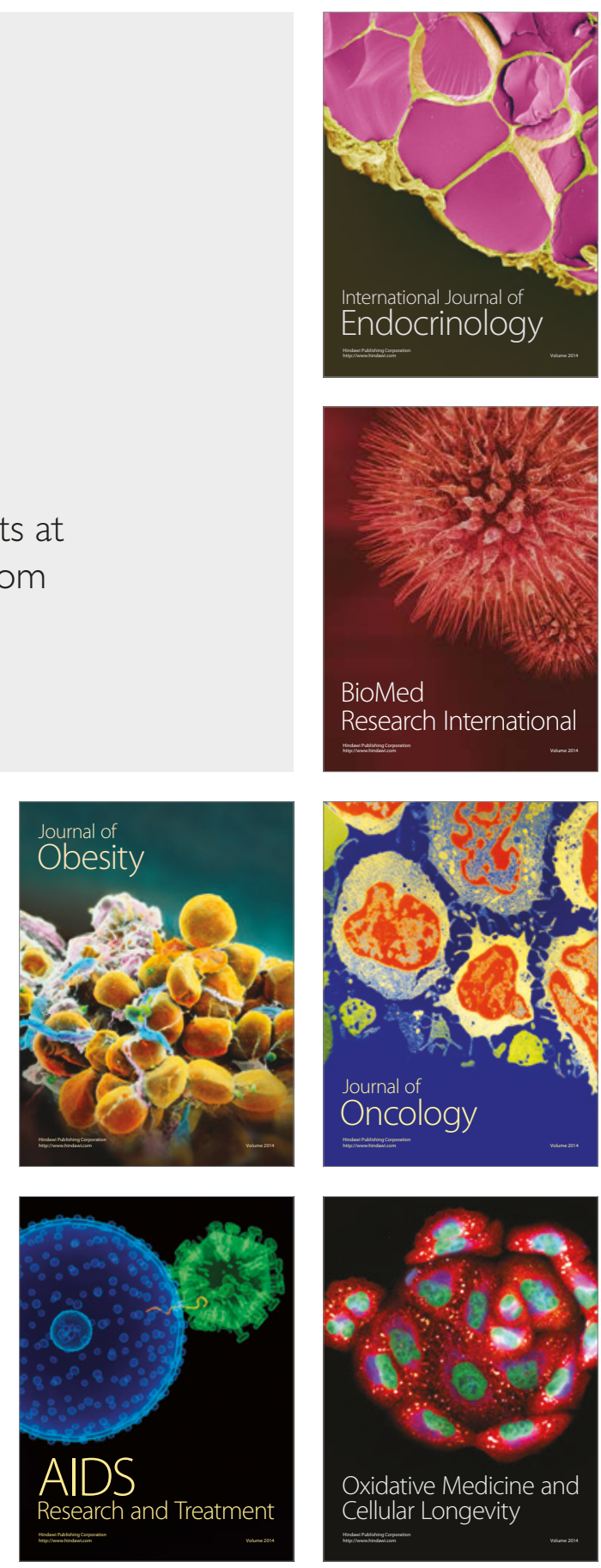